\title{
Modeling of parasitic effects in multi-rotor hybrid aircrafts (Part-II)
}

\author{
Ali Shahbaz Haider*, Ahsan Fayyaz, Fatima Ayoub, Samman Jabbar, Asfandyar Ghani
}

Department of Electrical Engineering, COMSATS Institute of IT, Wah, Pakistan

\section{ARTICLE INFO}

\section{Article history:}

Received 15 June 2016

Received in revised form

10 August 2016

Accepted 17 September 2016

\section{Keywords:}

Parasitic effects

Hybrid aircraft

T-Copter

Air drag

Tilt reaction

Coriolis acceleration

Reaction moments

Frictional moments

\section{Introduction}

A multi-rotor hybrid aircraft is a flying platform with more than one motor-propeller assemblies and it combines the flight features of the hovercraft and the wing aircraft. It can vertically take off and land (VTOL), and can hover in the air like helicopters (Haider and Sajjad, 2012). To track flight trajectory or to hover, the hybrid craft can change the configuration of the rotors using a tilt-wing mechanism, effectively transforming it from the wing craft configuration to the hovercraft configuration and vice versa (Partovi et al., 2011). It has many civilian and military applications (Kendoul et al., 2005). The development of the mini flying robots is flourishing for a couple of decades. The hybrid aircrafts are available in various configuration (Haider and Sajjad, 2012) and designs e.g. star, delta, mesh, cross structures (Goel et al., 2009). One of the most famous configurations is the Tee structure, known as T-Copter (Cruz et al., 2008). It is a challenging system to be modeled and controlled. The tiltrotor is a mechanism, which is used in most of the T-Copter to control the yaw. The objective of our research is to develop a VTOL based hybrid

\footnotetext{
* Corresponding Author.

Email Address: alishahbaz@ciitwah.edu.pk (A. S. Haider)

https://doi.org/10.21833/ijaas.2016.09.004

2313-626X/C 2016 The Authors. Published by IASE.

This is an open access article under the CC BY-NC-ND license

(http://creativecommons.org/licenses/by-nc-nd/4.0/)
}

aircraft (Zhang and Brandt, 1999), however, the multiple rotor-propeller sets produce complicated dynamic effects during hover as well as during maneuvers like roll, pitch and yaw. These effects result in the nonlinearities in the dynamics of the craft as well as introducing cross coupling in the dynamics (Johnson, 2012). These effects are called "parasitic" because they are the byproducts of the desired maneuvers during hover and flight. A sound understanding of these effects may enable us to mitigate or counterbalance them by appropriate structural configuration selection and the design modifications (Leishman, 2006). This research paper is part-II of the two-part research to model parasitic effects in the detail. Part-I (Haider et al., 2016) covered the detailed analysis of the parasitic precession moments and the gyroscopic moments. This part-II covers modeling of the parasitic effects that are generated in the T-Copter hybrid aircraft, during its typical maneuvers, including air drag moments, tilt reaction moment, angular acceleration effects, reaction moments and frictional moments. Some of these effects appear during transients in roll, pitch and yaw, while some may appear even during the steady state hover.

The organization of the paper is as follows: Section 2 describes the overview of the hybrid craft hardware and its dynamics in general. Section 3 describes the terms that account for the parasitic effects and presents the modeling of these effects. Section 4 deals with the modification of general 
dynamics of the hybrid aircraft to incorporate the effects of the parasitics that have been modeled in Section 3 and part-I of this paper. Section 5 presents the discussion and the conclusions.

\section{Overview of hardware and dynamics}

\subsection{Symbols and terminology}

Various symbols and terms that have been used to describe the dynamics of the T-copter are enlisted below:

$\mathbb{R}=$ Real Space.

$\phi=$ Roll, counter clockwise positive.

$\theta=$ Pitch, counter clockwise positive.

$\psi=$ Yaw, counter clockwise positive.

$\eta(\phi, \theta, \psi) \in \mathbb{R}^{3}=$ Angular displacement vector of the craft.

$M=$ mass of each rotor.

$\omega_{i}=$ angular velocity of the $i^{\text {th }}$ rotor.

$m=$ mass of entire hovercraft structure.

$\mathrm{I} \in \mathbb{R}^{3 \times 3}=$ Pseudo inertial matrix.

$\mathrm{R} \in \mathbb{R}^{3 \times 3}=$ Rotational matrix.

$\beta\left(E_{1}, E_{2}, E_{3}\right)=$ Body frame of reference.

$\Lambda\left(E_{x}, E_{y}, E_{z}\right)=$ Inertial frame of reference.

$C$. $G$. =Centre of gravity of the hovercraft body.

$\omega_{b}=$ Angular velocity of aircraft around $C$. $G$.

$\xi(x, y, z) \in \mathbb{R}^{3}=$ Position of the $C . G$. in $\Lambda$.

$l_{j}=$ Lengths of the respective sides of the hovercraft body.

$r_{j}=$ Distance of a point $j$ from the given axes of rotation.

$\mathrm{F} \in \mathbb{R}^{3}=$ Force vector in $\beta$.

$\tau \in \mathbb{R}^{3}=$ Torque vector.

$\mathrm{F}_{\mathrm{i}}=$ Force vector of the $i^{\text {th }}$ rotor.

$\aleph \in \mathbb{R}^{3}=$ Parasitic moment term in the system dynamics.

$\wp \in \mathbb{R}^{3}=$ Parasitic acceleration term in the system dynamics.

\subsection{System hardware}

The target system hardware is a tri-rotor hybrid aircraft as shown in Fig. 1. It can be considered a Tcopter rotorcraft with two main rotors and one tail rotor.

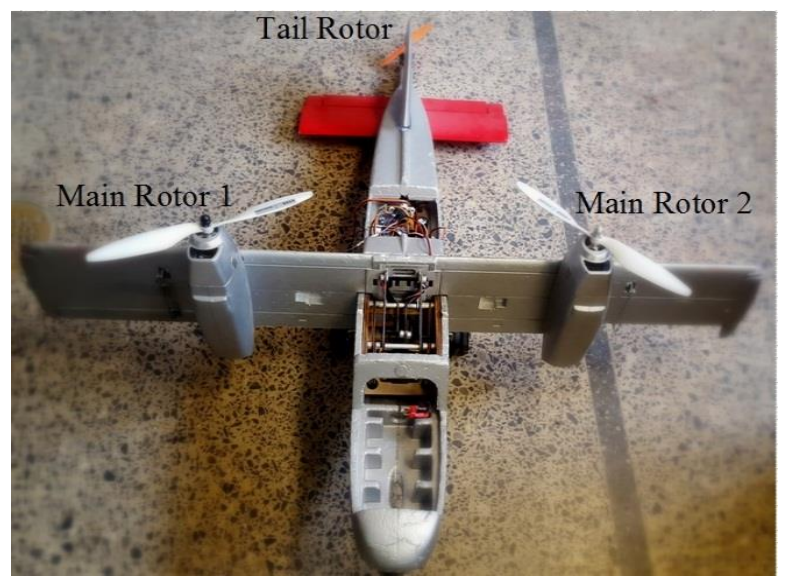

Fig. 1: The hybrid aircraft hardware
The tail rotor has a tilt rotor mechanism as shown in Fig. 2, which comprises of a servo motor that tilts a shaft coupled to the tail rotor, hence controlling the angle of inclination of the tail rotor. This mechanism controls the yaw and compensates some of the parasitic effects, as it will be explained in the further sections.

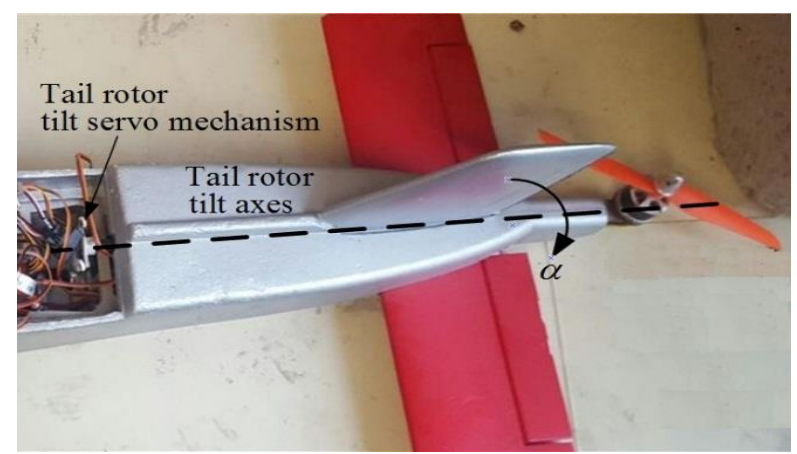

Fig. 2: The tail rotor tilt mechanism

The tilt wing mechanism is shown in Fig. 3. It transforms the aircraft structure form the hover mode to the flight mode and vice versa, using a servo mechanism similar to the tilt rotor mechanism.

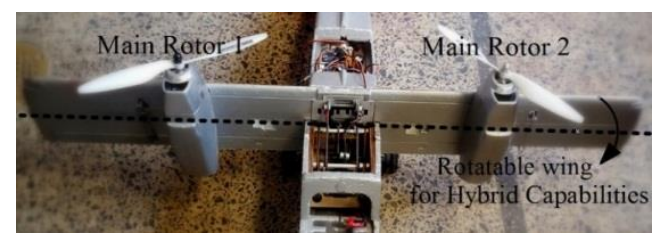

Fig. 3: The tilt-wing mechanism

A simplified T-Copter structure for the hardware modeling is shown in Fig. 4. The frames of references and reference directions of rotations of various rotors are also shown in Fig. 4. It is worth noting that the two main rotors rotate in opposite directions to cancel some of the parasitic effects as explained in further sections. The tilt rotor mechanism makes two orthogonal components of the force $\mathbf{F}_{3}$ in $E_{2} E_{3}$ plane as shown in Fig. 5.

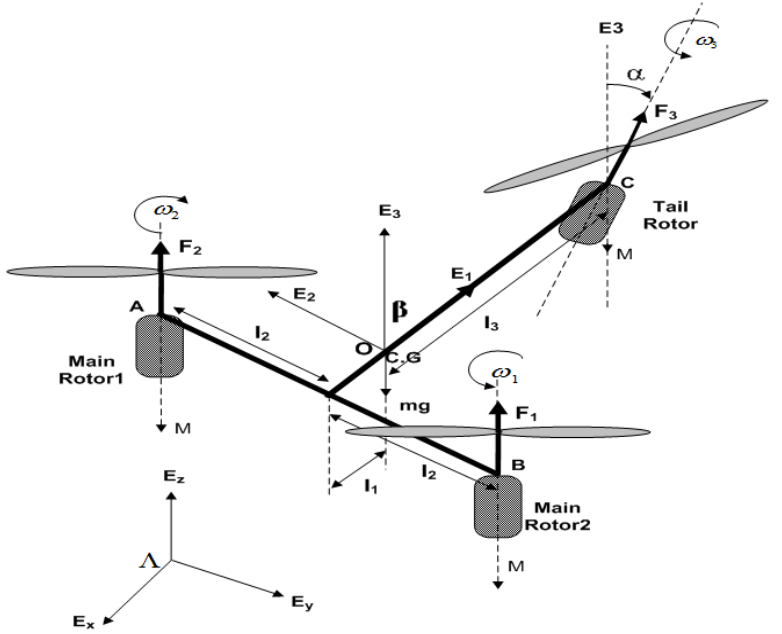

Fig. 4: Simplified T-Copter Structure for system modeling

The Newton-Euler's equations govern the dynamics of any general aircraft (Haider and Sajjad, 2012). These equations describe the dynamics of the 
body of the aircraft in the inertial frame of reference and these equations are given by:

$$
\begin{aligned}
& m \frac{d^{2} \xi}{d t^{2}}=\mathrm{RF}-m g \mathrm{E}_{\mathrm{z}}+\wp\left(\omega_{j}, r_{i}, \xi, \eta\right) \\
& \mathrm{I} \frac{d^{2} \eta}{d t^{2}}=\tau+\aleph\left(\omega_{j}, l_{k}, r_{i}, \eta\right)
\end{aligned}
$$

where, $\mathrm{E}_{\mathrm{z}}=\left[\begin{array}{lll}0 & 0 & 1\end{array}\right]^{\mathrm{T}}$.

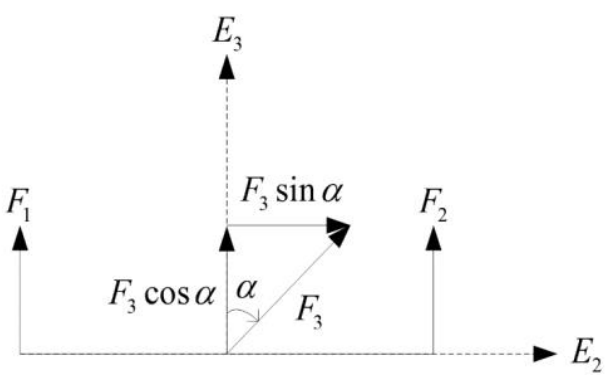

Fig. 5: Components of $\mathrm{F}_{3}$ in $E_{2} E_{3}$-plane

\section{Parasitic effects in aircraft dynamics}

The terms in the vectors $\wp$ and the vector $\aleph$ in Eq.1 correspond to the parasitic accelerations and the parasitic moments respectively. Various parasitic effects can be categorized as follows:

1. The precession moments.

2. The gyroscopic moments.

3. The air drag moments.

4. The angular acceleration effects.

5. Reaction moments.

6. Frictional moments.

The parasitic moment term $\aleph$ can be decomposed into three terms for this research paper as given by Eq. 2.

$$
\kappa=\aleph_{p}+\kappa_{g}+\kappa_{m}
$$

where,

1. $\aleph_{\mathbf{p}}=$ Parasitic precession moments

2. $\aleph_{\mathrm{g}}=$ Parasitic gyroscopic moment.

3. $\aleph_{\mathrm{m}}=$ Parasitics other than $\aleph_{\mathrm{p}}$ and $\aleph_{\mathrm{g}}$.

In part-I of this research work, the precession and gyroscopic parasitic effects have been presented. This part of the research work presents terms included in $\aleph_{m}$, namely air drag moments, frictional moments, angular acceleration effects and reaction moments.

\subsection{Air drag moments}

When the blades of the propeller rotate, they drag through the air resulting in air drag moments around the spin axis of the propeller. The direction of these moments is counter to the direction of rotation of the propellers. This moment is a function $\mathrm{p}(\cdot)$ of the angular speed of the propeller. The air drag moment of the $i^{\text {th }}$ propeller, $\mathrm{Q}_{\mathrm{i}}$ can be expressed by Eq. 3 .

$$
\mathrm{Q}_{\mathrm{i}}=\mathrm{p}\left(\omega_{\mathrm{i}}\right)
$$

The function $\mathrm{p}(\cdot)$ is typically a polynomial function. Its order and coefficients are determined by the curve fitting techniques to the experimental data that maps the drag moment for the angular velocity of the propeller. The air drag moment vectors for the individual propeller are shown in Fig. 6. There expression is given by Eq. 4 .

$$
\begin{aligned}
& \mathrm{Q}_{1}=\left[\begin{array}{lll}
0 & 0 & -\left|\mathrm{Q}_{1}\right|
\end{array}\right]^{\mathrm{T}} \\
& \mathrm{Q}_{2}=\left[\begin{array}{lll}
0 & 0 & \left|\mathrm{Q}_{2}\right|
\end{array}\right]^{\mathrm{T}} \\
& \mathrm{Q}_{3}=\left[\begin{array}{lll}
0 & \left|\mathrm{Q}_{3}\right| \sin \alpha & -\left|\mathrm{Q}_{3}\right| \cos \alpha
\end{array}\right]^{\mathrm{T}}
\end{aligned}
$$

The net air drag moment $M_{a d}$ is the sum of the individual moments in Eq. 4 and is given by Eq. 5 .

$$
\mathrm{M}_{\mathrm{ad}}=\sum_{i=1}^{3} \mathrm{Q}_{\mathrm{i}}=\left[\begin{array}{c}
0 \\
\left|\mathrm{Q}_{3}\right| \sin \alpha \\
-\left|\mathrm{Q}_{1}\right|+\left|\mathrm{Q}_{2}\right|-\left|\mathrm{Q}_{3}\right| \cos \alpha
\end{array}\right]
$$

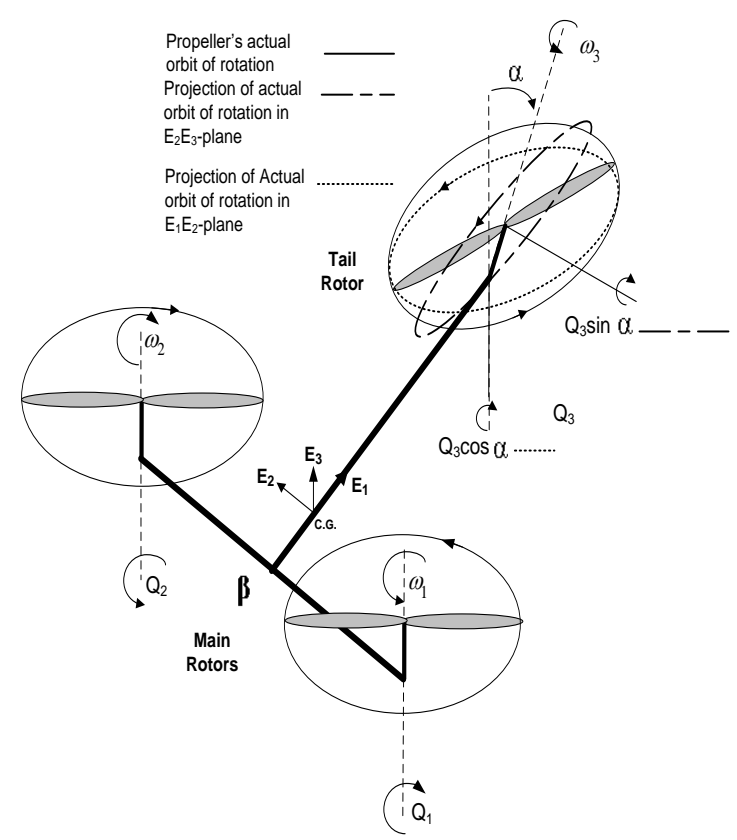

Fig. 6: Air drag moments for the T-Copter propellers

Since $\omega_{1}$ and $\omega_{2}$ are opposite in direction so they neutralizing the air drag effects $Q_{1}$ and $Q_{2}$ to the great extent for the craft body. Its drag moment component $\left|Q_{3}\right| \sin \alpha$ acts as a disturbance in the pitch controlling feedback loop and it has to be rejected. The other component $-\left|Q_{3}\right| \cos \alpha$ is partially balanced by the torqueing effect of component of the force vector $\mathrm{F}_{3}$ in $\mathrm{E}_{1} \mathrm{E}_{2}$-plane in Fig. 5 that is $l_{3} F_{3} \sin \alpha$. These moments shift their axes of application from the spin axis of the propellers to C.G. of the structure (aerodynamic centre of gravity) in accordance with Varignon's Second Moment Theorem, as shown in Fig. 7.

The static Air drag calibration is done using laboratory setup shown in Fig. 8. The actuating signal to the motor driver board is varied and the upward thrust is balanced by counter weight $\mathrm{W}$. The sideways air drag pulls the thread, resulting in a change in the reading of the electronic balance, proportional to the air drag of the propeller. 
The second order and third order air drag calibration curves are shown in Fig. 9. A third order curve fit is given by Eq. 6 .

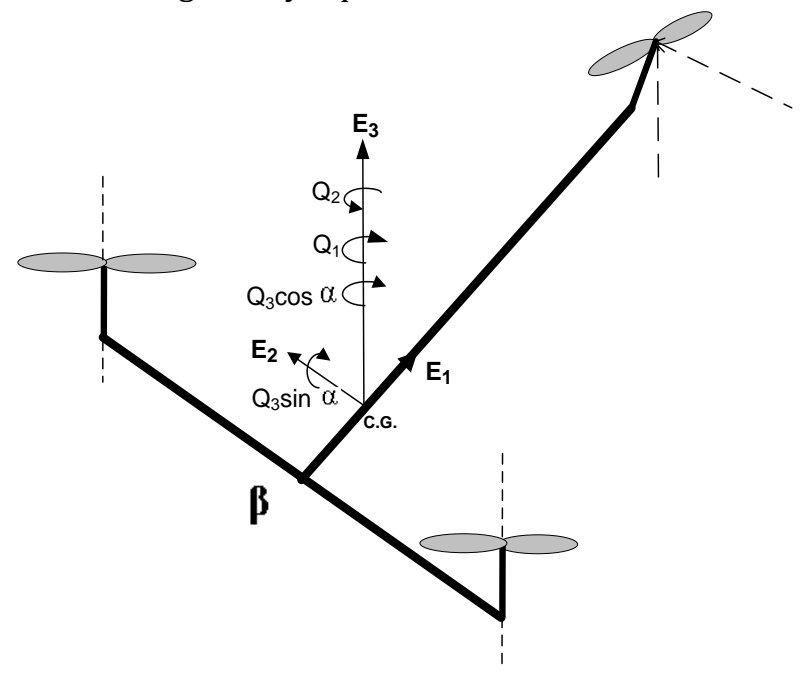

Fig. 7: Air drag moments shifting axes to C.G.

$$
\begin{aligned}
& \left|\mathbf{Q}_{\mathrm{i}}\right|=4.21 \times 10^{-8} u_{i}^{3}-1.27 \times 10^{-5} u_{i}^{2}+1.58 \times \\
& 10^{-8} u_{i}-0.031
\end{aligned}
$$

where, $u_{i} \in(0.06,0.085)$

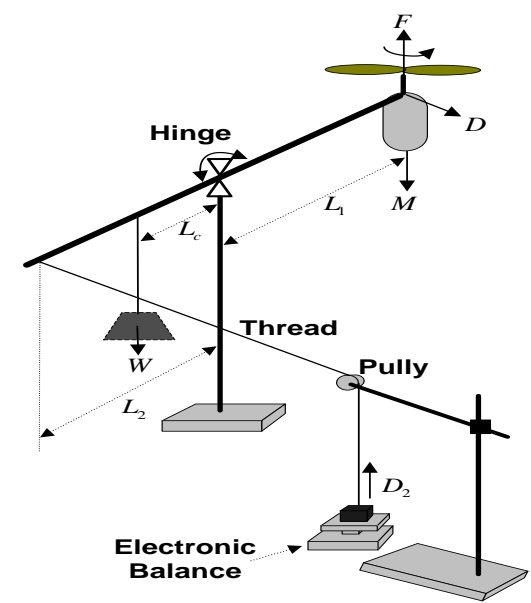

Fig. 8: Static Air drag calibration setup

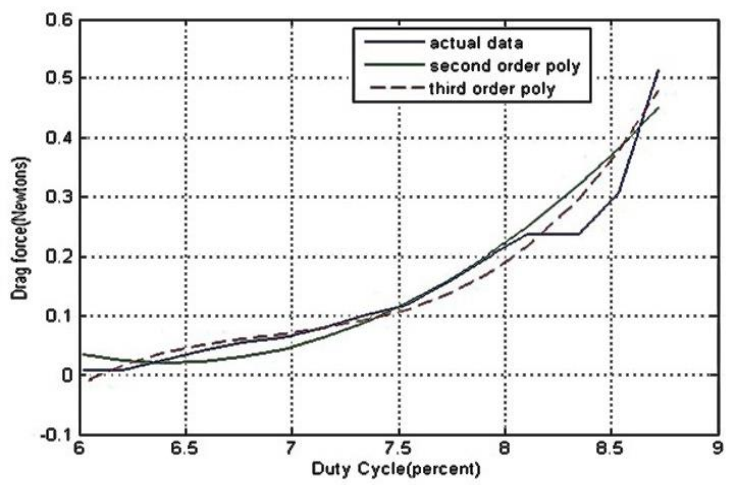

Fig. 9: Static air drag calibration curves

\subsection{The reaction moments}

As the tail rotor tilts in response to the commanded value of the angle $\alpha$, a counter moment is produced on the aircraft body according to Newton's third Law, hence the name tilt reaction moment, denoted by $\mathrm{M}_{\mathrm{tr}}$. It is shown in Fig. 10. The tilt reaction moment acts around $\mathrm{E}_{1}$-axis. If the moment of inertia of the structure around $E_{1}$-axis is given by $I_{t}$, then the tilt reaction moment vector is given by Eq. 7 .

$$
\mathrm{M}_{\mathrm{tr}}=\left[\begin{array}{c}
-I_{t} \ddot{\alpha} \\
0 \\
0
\end{array}\right]
$$

Moreover as the speeds of rotation of the propellers change, reaction effects are produced in accordance with the Newton's third law. These effects are denoted by $\mathrm{M}_{\mathrm{r}}$, and are given by Eq. 8 .

$$
\mathrm{M}_{\mathrm{r}}=\left[\begin{array}{c}
0 \\
\dot{\omega}_{3} \sin \alpha \\
\dot{\omega}_{1}-\dot{\omega}_{2}-\dot{\omega}_{3} \cos \alpha
\end{array}\right]
$$

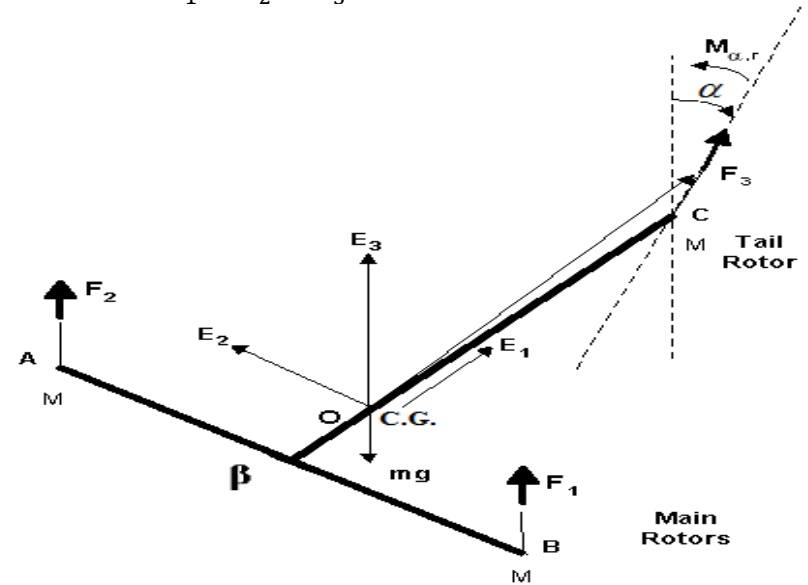

Fig. 10: Tilt reaction moment

\subsection{The angular acceleration moments}

Consider Fig. 11 in which a particle at a position denoted by point $\mathrm{P}$, moves along a path on a lamina. Various terms are given by,

$B=$ the base point of pole point $C . G$.

$X Y Z$-frame=the fixed reference frame with the origin $O$

$x y z$-frame=the fixed body frame rotating about $\mathrm{z}$ axis or Z-axis.

$\omega_{b}=$ the angular velocity of the lamina along $\mathrm{Z}$ or z-axis

$\mathrm{R}=$ the position vector of the $C . G$. relative to the $X Y Z$ frame

$\mathrm{r}=$ the position vector of $\mathrm{P}$ relative to the $X Y Z$ frame

$\rho=$ the position vector of $\mathrm{P}$ relative to the xyz frame $\mathrm{a}_{\mathrm{P}}=$ the total acceleration of $\mathrm{P}$

$\mathrm{a}_{\mathrm{p} \text {,path }}=$ the acceleration of $\mathrm{P}$ relative to the path, considering the path stationary

$\mathrm{a}_{\mathrm{M}}$ =the acceleration of the point $\mathrm{M}$ on lamina whose position coincides with the point $\mathrm{P}$ at any given instant.

$\mathrm{a}_{\text {cor }}=$ Coriolis component of acceleration $\mathrm{d}=$ deceleration

If the lamina itself moves and rotates then the various accelerations produced in the particle at position $\mathrm{P}$ are shown in Fig. 12 . The subscripts $n$ 
and $t$ denote normal and tangential components respectively and the subscriptstrans androt denote the translational and the rotational components respectively.

The total acceleration of point $\mathrm{P}$ is given by Eq. 9.

$$
a_{p}=a_{c o r}+a_{M}+a_{P, p a t h}
$$

If $\mathrm{v}_{\mathrm{P}, \text { path }}$ denote the tangential velocity of $\mathrm{P}$ in xyz- frame then the coriolis acceleration is given by Eq. 10.

$$
\mathrm{a}_{\text {cor }}=2 \omega_{\mathrm{b}} \times \mathrm{v}_{\mathrm{P}, \text { path }}=2\left|\omega_{\mathrm{b}}\right|\left|\mathrm{v}_{\mathrm{P}, \text { path }}\right| \sin (\Lambda) \hat{\mathrm{k}}
$$

here $\Lambda$ is the angle between $v_{P, p a t h}$ and $\omega_{b}$, and $\hat{k}$ is the unit vector normal to plane of the aforementioned two vectors.

The term $a_{M}$ in Eq. 9 can be decomposed into translational and rotational components as given by Eq. 10.

$$
\mathrm{a}_{\mathrm{M}}=\mathrm{a}_{\mathrm{M}, \text { trans }}+\mathrm{a}_{\mathrm{M}, \mathrm{rot}}
$$

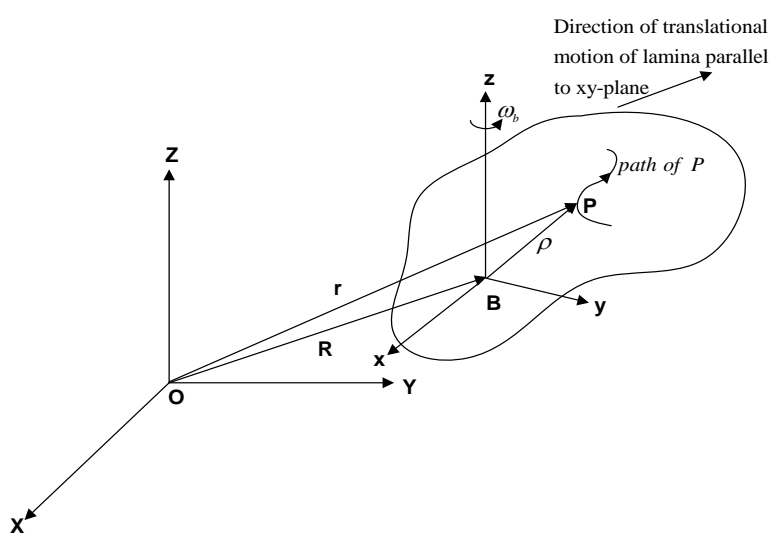

Fig. 11: Motion of a particle on a moving lamina

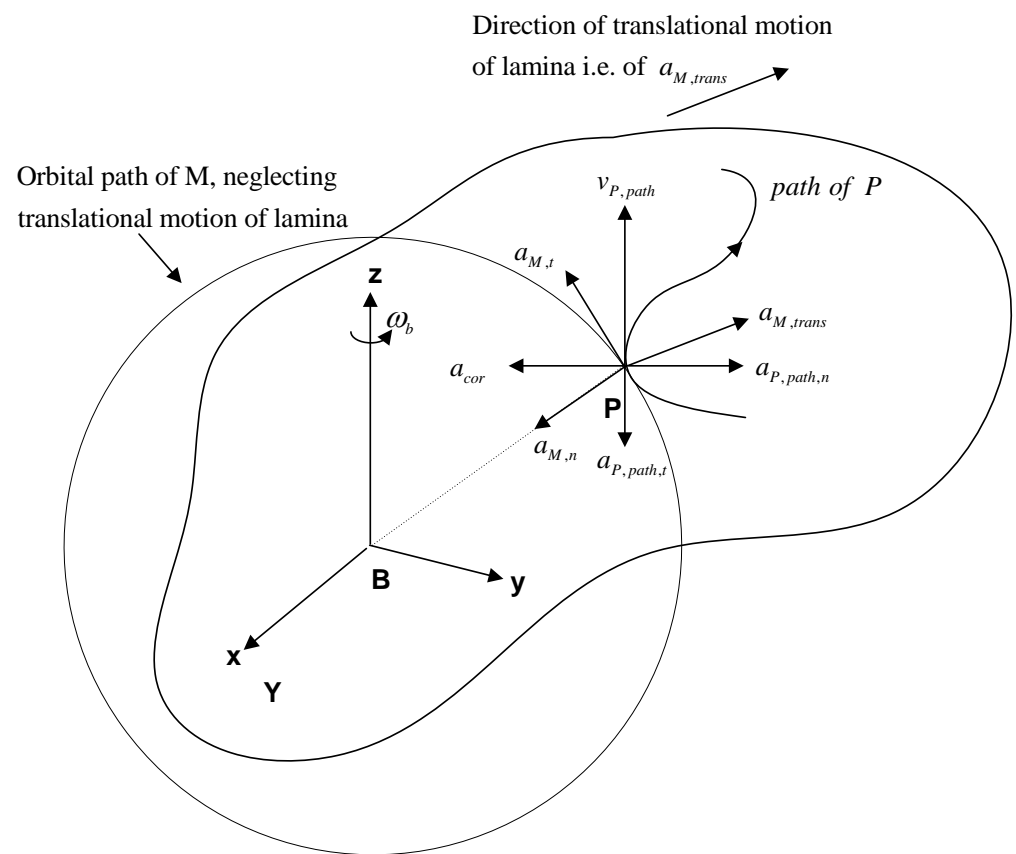

Fig. 12: Various accelerations produced on a particle $\mathrm{P}$ on a moving lamina

The rotational component of $a_{M}$ can be decomposed into normal and tangential components as follows,

$$
\mathrm{a}_{\mathrm{M}, \mathrm{rot}}=\mathrm{a}_{\mathrm{M}, \mathrm{n}}+\mathrm{a}_{\mathrm{M}, \mathrm{t}}
$$
13.

The rotational component of $\mathrm{a}_{\mathrm{M}}$ is given by Eq.

$$
\begin{aligned}
& \left|\mathrm{a}_{\mathrm{M}, \mathrm{t}}\right|=\frac{d^{2} s}{d t^{2}}=\frac{d^{2}(\rho \theta(t))}{d t^{2}}=\rho \dot{\omega}_{b} \\
& \left|\mathrm{a}_{\mathrm{M}, \mathrm{n}}\right|=\frac{1}{\rho}\left(\frac{d s}{d t}\right)^{2}=\rho \omega_{b}^{2}
\end{aligned}
$$

If $\mathbf{F}_{\text {net }}$ is the net force on the lamina, having a mass $m_{t}$ then the translational component of $\mathrm{a}_{\mathrm{M}}$ is given by Eq. 14 .

$$
\left|\mathrm{a}_{\mathrm{M}, \text { trans }}\right|=\frac{\left|\mathrm{F}_{\text {net }}\right|}{m_{t}}
$$

The acceleration $a_{P, p a t h}$ in Eq. 9 can be decomposed into tangential and normal components as given by Eq. 15 .

$$
\mathrm{a}_{\mathrm{P} \text {,path }}=\mathrm{a}_{\mathrm{P} \text {,path,t }}+\mathrm{a}_{\mathrm{P} \text {,path, }}
$$

The components of $\mathrm{a}_{\mathrm{P} \text {,path }}$ in Eq. 9 are mathematically expressed by Eq. 16 .

$$
\begin{aligned}
& \left|\mathrm{a}_{\mathrm{P}, \text { path,t }}\right|=\gamma\left(\left|\mathrm{v}_{\mathrm{P}, \text { path }}\right|^{2}\right) \\
& \left|\mathrm{a}_{\mathrm{P}, \text { path,n }}\right|=\left|\dot{\mathrm{v}}_{\mathrm{P}, \text { path }}\right|
\end{aligned}
$$

here $\gamma$ is the radius of curvature of the path of the particle $\mathrm{P}$ on the lamina. The net acceleration of particle $\mathrm{P}$ in Fig. 12 is elucidated using vector addition technique in Fig. 13.

Now the above formulation of Eq. 9 through Eq. 16 is used for the case of the propeller blade. Let the rotorcraft be parallel to the XY-plane. Let $\mathrm{P}_{1}$ and $\mathrm{P}_{2}$ 
be any of the two points on the two opposite sides of propeller blades at a distance $\rho$ from the axes of rotation C. The Fig. 14 shows the acceleration analysis for these two points on the propeller blades as the propellers rotate, assuming zero aircraft $\operatorname{velocity}\left(\omega_{b}\right)$.

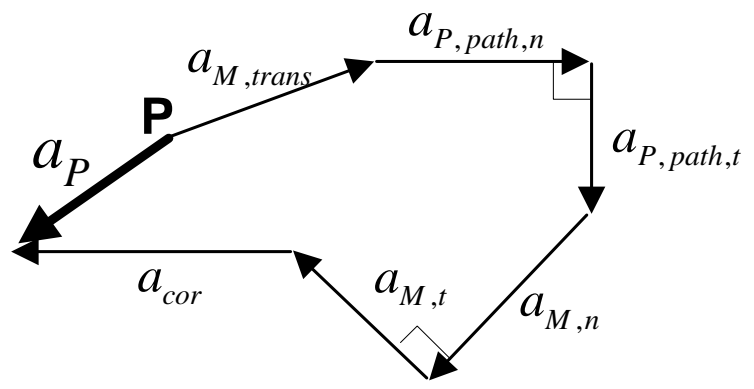

Fig. 13: Net acceleration of the particle $P$ by vector addition

For the case of the nonzero velocity of the aircraft body, let points $M_{1}$ and $M_{2}$ on the propeller blades move in the radius of circles $r_{1}$ and $r_{2}$ respectively. The net acceleration for a given point $P_{i}$, for $a$ particular scenario is shown in Fig. 15. The circular trajectories traversed by the M-points are also shown in Fig. 15. Following the development of Eq. 9, in general, for any point $\mathrm{P}_{\mathrm{i}}$ we have,

$$
\begin{aligned}
& \mathrm{a}_{\mathrm{P}_{\mathrm{i}}}=\mathrm{a}_{\mathrm{M}_{\mathrm{i}}, \mathrm{r}_{\mathrm{i}}}+\mathrm{a}_{\mathrm{M}, \text { trans }}+\mathrm{a}_{\mathrm{i}} \\
& \mathrm{a}_{\mathrm{M}_{\mathrm{i}}, \mathrm{r}_{\mathrm{i}}}=\mathrm{a}_{\mathrm{M}_{\mathrm{i}}, \mathrm{t}}+\mathrm{a}_{\mathrm{M}_{\mathrm{i}}, \mathrm{n}} \\
& \mathrm{a}_{\mathrm{i}}=\mathrm{a}_{\text {cor }}+\mathrm{a}_{\mathrm{P}_{\mathrm{i}, \text { path }, \mathrm{t}}}+\mathrm{a}_{\mathrm{P}_{\mathrm{i}}, \text { path,n }}
\end{aligned}
$$

displacement of the propeller during its rotation and for any value of $\rho$. The accelerationsa ${ }_{1}$ anda $_{2}$ always make a specific couple as shown in the Fig. 16. The couple keeps rotating with the propeller, however the angle $\chi$ remain the same. For $\chi=0$, the perfect cancellation of this couple takes place.

The acceleration component $a_{M_{i}, r_{i}}$ in Eq. 17 is dependent upon the values of $\omega_{i}, \dot{\omega}_{b}$ and $r_{i}$ as given by Eq. 18.

$$
\begin{aligned}
& \left|\mathrm{a}_{\mathrm{M}_{\mathrm{i}}, \mathrm{n}}\right|=r_{i} \omega_{b}^{2} \\
& \left|\mathrm{a}_{\mathrm{M}_{\mathrm{i}}, \mathrm{t}}\right|=r_{i} \dot{\omega}_{b}
\end{aligned}
$$

The normal and tangential components of $\mathbf{a}_{\mathbf{M}_{\mathbf{i}}, \mathbf{r}_{\mathbf{i}}}$ are shown in Fig. 17.

If we consider the points $C$. $G$.and $C$ as shown in the Fig. 18 then the points $P_{i}$ can be expressed by Eq. 19 and $r_{i}$ is given by Eq. 20 .

$$
\begin{aligned}
& \mathrm{P}_{1}=\left[\begin{array}{l}
x+\rho \cos (v) \\
y+\rho \sin (v)
\end{array}\right] \\
& \mathrm{P}_{2}=\left[\begin{array}{l}
x-\rho \cos (v) \\
y-\rho \sin (v)
\end{array}\right] \\
& \left\|\mathrm{P}_{1}\right\|_{2}=r_{1}=\sqrt[2]{(x+\rho \cos (v))^{2}+(y+\rho \sin (v))^{2}} \\
& \left\|\mathrm{P}_{2}\right\|_{2}=r_{2}=\sqrt[2]{(x-\rho \cos (v))^{2}+(y-\rho \sin (v))^{2}}
\end{aligned}
$$

The simulation results for the values of $r_{i}$ with variation in $v$ and $\rho$ are plotted in Fig. 19 as a 3-D surface. The example simulation values used are: $x=0.5, y=0.5, v \in(0,2 \pi)$ and $\rho \in(0,0.45)$.

here $\left|a_{i}\right|$ remains the same for any two opposite points $\mathrm{P}_{\mathrm{i}}$ on the propeller blades, for any circular

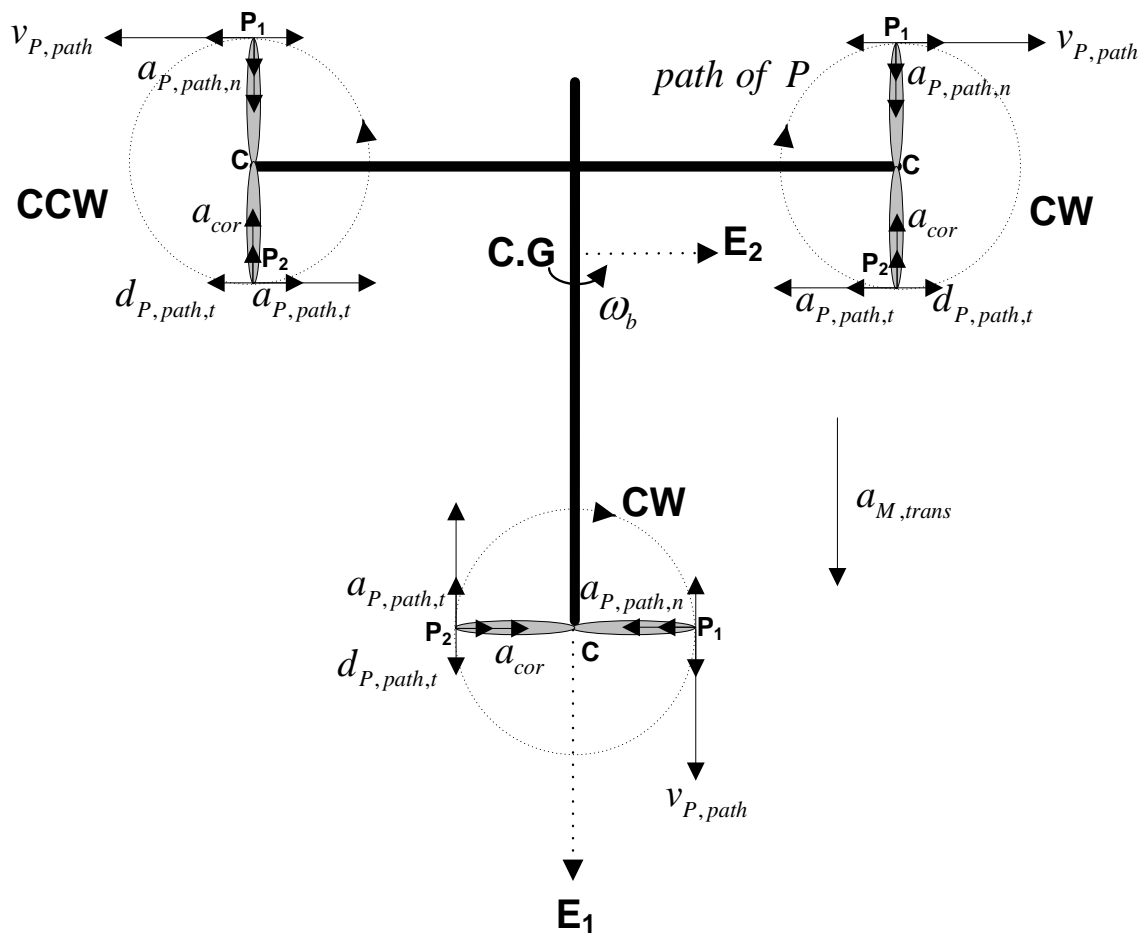

Fig. 14: Various accelerations for the blade elements 


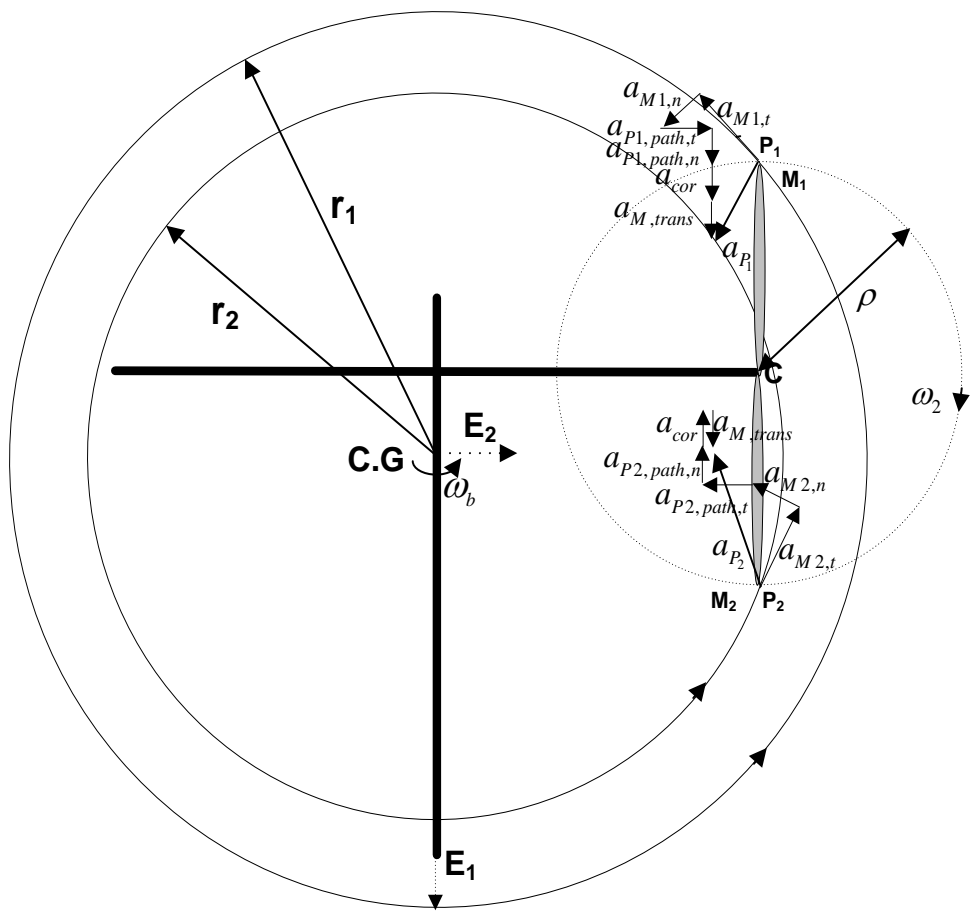

Fig. 15: Net Accelerations of the blade elements

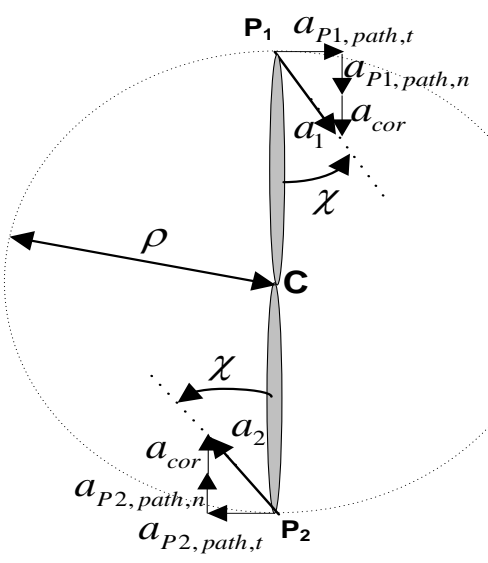

Fig. 16: A specific couple made by $\mathrm{a}_{1}$ and $\mathrm{a}_{2}$

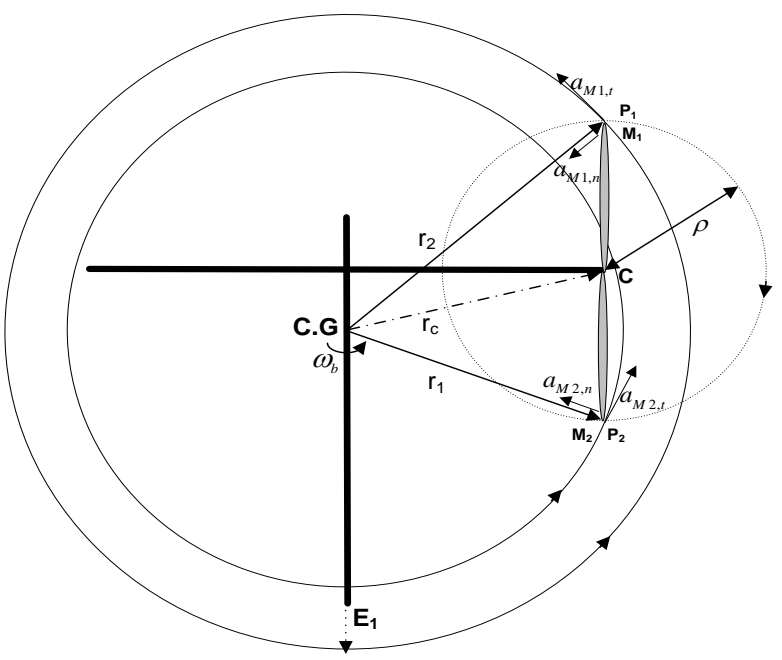

Fig. 17: Normal and tangential components of $a_{M_{i}, r_{i}}$

The 2-D perspective of Fig. 19 illustrating the variations of $r_{i}$ with a fixed $\rho=0.25$ and varying $v$ in the interval $(0,2 \pi)$ are shown in Fig. 20. The 2-D perspective of Fig. 19 illustrating the variations of $r_{i}$ with a fixed $v=\pi / 4$ and varying $\rho$ in interval $(0,0.45)$ are shown in Fig. 21.

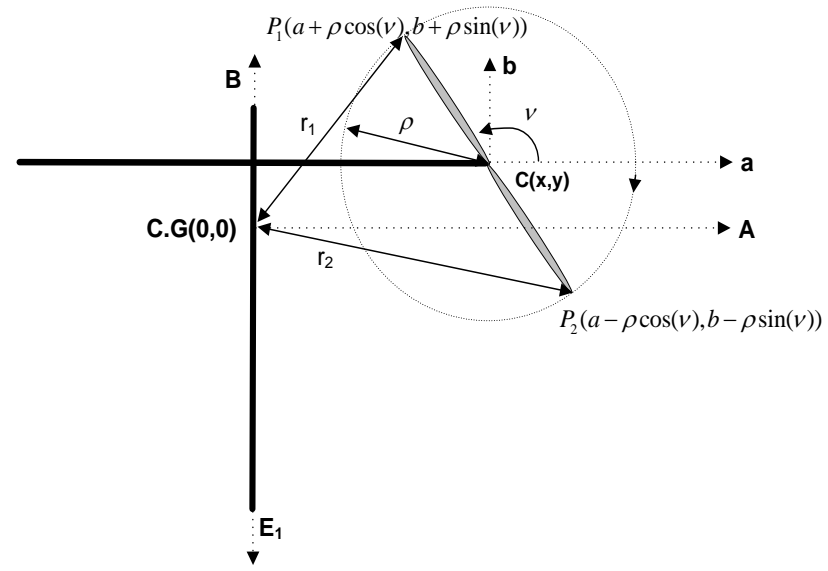

Fig. 18: Position vectors of points $P_{i}$

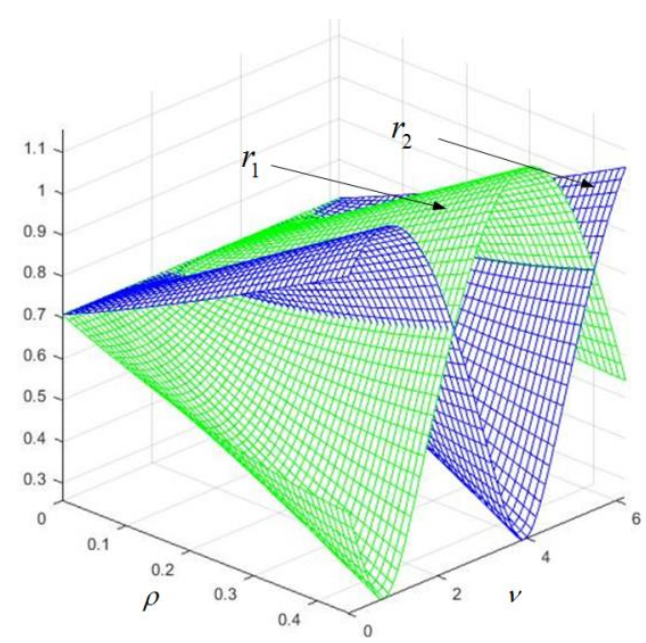

Fig. 19: Variation of $r_{i}$ with $\rho$ and $v$ 


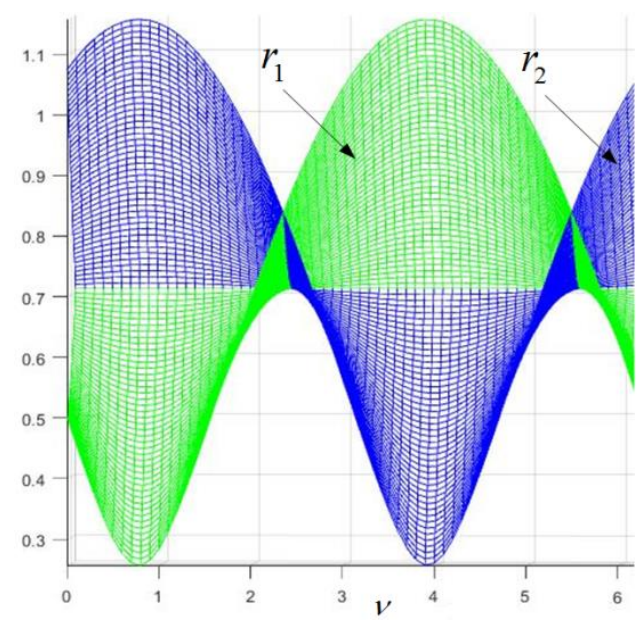

Fig. 20: Variation of $r_{i}$ with $v$

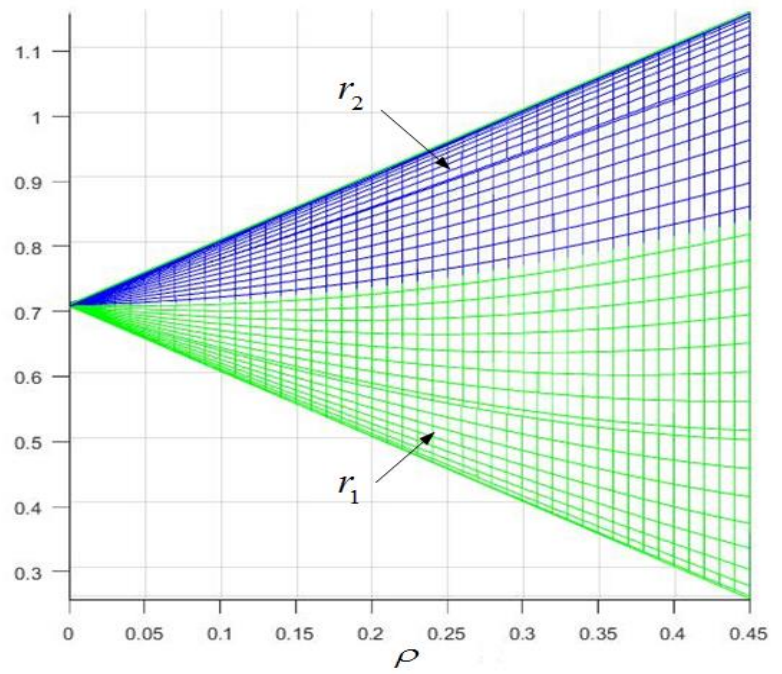

Fig. 21: Variation of $r_{i}$ with $\rho$

Frictional effects: The frictional moments experienced by the aircraft body are given by Eq. 21 .

$\mathrm{M}_{\mathrm{f}}=k_{f m}\left[\begin{array}{c}\dot{\phi} \\ \dot{\theta} \\ \dot{\psi}\end{array}\right]$

The frictional forces experienced by the aircraft body are given by Eq. 22 .

$$
\mathrm{F}_{\mathrm{f}}=-k_{f F}\left[\begin{array}{c}
\dot{x} \\
\dot{y} \\
\dot{z}
\end{array}\right]
$$

\section{Dynamics equations including parasitics}

The term $\aleph_{m}$ that accounts for parasitic effects in Eq. 2 can now be expressed by Eq. 23.

$$
\aleph_{\mathrm{m}}=\mathrm{M}_{\mathrm{ad}}++\mathrm{M}_{\mathrm{r}}+\mathrm{M}_{\mathrm{tr}}+\mathrm{M}_{\mathrm{f}}
$$

The term $\wp$ that accounts for parasitic effects in Eq. 1 can now be expressed by Eq. 24 .

$$
\wp=F_{f}+F_{\text {prop }}
$$

Here $\mathrm{F}_{\text {prop }}$ includes the parasitic forces results from the accelerations on the propeller blade elements from Eq. 17. By including the parasitic effects, the system in Eq. 1 modifies to Eq. 25,

$$
\begin{aligned}
& m \frac{d^{2} \xi}{d t^{2}}=\mathrm{RF}-m g \mathrm{E}_{\mathrm{z}}+\mathrm{F}_{\mathrm{f}}+\mathrm{F}_{\text {prop }} \\
& \mathrm{I} \frac{d^{2} \eta}{d t^{2}}=\tau+\aleph_{\mathrm{p}}+\aleph_{\mathrm{g}}+\mathrm{M}_{\mathrm{ad}}+\mathrm{M}_{\mathrm{tr}}+\mathrm{M}_{\mathrm{r}}+\mathrm{M}_{\mathrm{f}}
\end{aligned}
$$

It is clear that the model in Eq. 25 is not only nonlinear but also has cross coupling owing to parasitic effects.

\section{Discussion and conclusions}

The dynamics of multi-rotor hybrid aircraft have been reviewed, taking into the account the terms that include the undesirable parasitic effects. Amongst various parasitic effects, air drag moments, tilt reaction moment, angular acceleration effects, reaction moments and frictional moments have been modeled in the detail. The developed equations have been elucidated and the complete model of the aircraft that includes all of the parasitics has been presented. It has been shown that the parasitic terms are the major source of nonlinearities and cross coupling in the dynamics of the aircraft.

\section{References}

Cruz SS, Kendoul F, Lozano R and Fantoni I (2008). Real time stabilization of a small three rotor aircraft. IEEE Transactions on Aerospace and Electronic Systems, 44(2): 783-793

Goel R, Shah SM, Gupta NK and Ananthkrishnan N (2009). Modeling, simulation and flight testing of an autonomous quadrotor. Proceedings of ICEAE, Indian Institute of Science, India.

Haider AS and Sajjad M (2012). Structural Design and Non-linear Modeling of a Highly Stable MultiRotor Hovercraft. Control Theory and Informatics, 2(4): 24-35.

Haider AS, Ali R, Aftab Q, Asim M, and Akram T (2016). Modeling of parasitic effects in multirotor hybrid aircrafts: Part-I. International Journal of Advanced and Applied Sciences, 3(7):11-17

Johnson W (2012). Helicopter Theory. Courier Corporation. North Chelmsford, USA.

Kendoul F, Fantoni I and Lozano R (2005). Modeling and control of a small autonomous aircraft having two tilting rotors. The European Control Conference, Seville, Spain: 8144-8147

Leishman JG (2006). Principles of Helicopter Aerodynamics. Cambridge University Press. Cambridge, UK.

Partovi AR, Xinhua W, Lum KY and Hai L (2011). Modeling and control of a small-scale hybrid 
Haider et al/International Journal of Advanced and Applied Sciences, 3(9) 2016, Pages: 16-24

aircraft. IFAC Proceedings Volumes, 44(1): 10385-10390
Zhang W and Brandt RD (1999). Robust hovering control of PVTOL aircraft. IEE Transactions on Control Systems Technologies, 7(3): 343-351. 